\title{
A Knowledge Comparison Environment for Supporting Meaningful Learning of E-Book Users $†$
}

\author{
Jingyun Wang ${ }^{1, *}$, Hiroaki Ogata ${ }^{2}$ and Atsushi Shimada ${ }^{2}$ \\ 1 Research Institute for Information Technology, Kyushu University, Fukuoka 812-8581, Japan \\ 2 Faculty of Arts and Science, Kyushu University, Fukuoka 819-0395, Japan; \\ ogata@artsci.kyushu-u.ac.jp (H.O.); atsushi@limu.ait.kyushu-u.ac.jp (A.S.) \\ * Correspondence: warmplam@gmail.com or wang.jingyun.579@m.kyushu-u.ac.jp; Tel.: +81-092-642-2297 \\ + This paper is an extended version of our paper published in the 23rd International Conference on \\ Computers in Education (ICCE 2015), Hangzhou, China, 30 November-4 December 2015
}

Academic Editor: Jon Mason

Received: 31 January 2016; Accepted: 28 April 2016; Published: 16 May 2016

\begin{abstract}
In this paper, we present an ontology-based visualization support system which can provide a meaningful learning environment to help e-book learners to effectively construct their knowledge frameworks. In this personalized visualization support system, learners are encouraged to actively locate new knowledge in their own knowledge framework and check the logical consistency of their ideas for clearing up misunderstandings; on the other hand, instructors will be able to decide the group distribution for collaborative learning activities based on the knowledge structure of learners. For facilitating those visualization supports, a method to semi-automatically construct a course-centered ontology to describe the required information in a map structure is presented. To automatically manipulate this course-centered ontology to provide visualization learning supports, a prototype system is designed and developed.
\end{abstract}

Keywords: meaningful learning; critical thinking; knowledge structure; comparison skill; course-centered ontology

\section{Introduction}

Nowadays, e-book systems are widely used together with Learning Management Systems in the education field. For example, in the Arts and Science Department of Kyushu University Faculty, not only Moodle but also the BookLooper e-book system developed by Kyocera Communication Systems is used for supporting daily classroom teaching. Those two systems provide a platform for instructors to easily organize and manage teaching materials; on the other hand, learners can conveniently browse the learning resources. Especially, in the BookLooper system learners can even mark text or add comments on any PDF files and the system can record their learning activities (including which pages they read and how they switch between pages) and report to instructors [1]. However, as other existing systems, neither BookLooper nor Moodle provide any function to support learners in constructing their knowledge framework effectively. When a learner acquires different knowledge items, she/he may also compare those items and understand some relations between them at the same time; those acquired knowledge items and their relations form the knowledge framework of the learner. Knowledge framework development usually is not supported in the older systems, and also, in those systems it is difficult to identify the relevant knowledge items that a learner possesses before and after a learning activity.

Ausubel's learning psychology theories [2-4] define the effective assimilation of new knowledge into an existing knowledge framework as the achievement of "meaningful learning". Those theories suggest that knowledge finally gets incorporated into the human brain when organized in hierarchical 
frameworks and that learning approaches that facilitate this kind of organization significantly enhance the learning capability of all learners. In contrast, in the case of rote learning, knowledge tends to be quickly forgotten unless rehearsed repeatedly. Moreover, retained knowledge cannot contribute to enhance the learner's knowledge framework and has a low possibility of being used in future problem-solving [5]. However, how to move beyond rote learning and help learners to effectively construct their framework is still an open issue.

In our previous research [6,7], the experimental results suggest that with the support of a system, which provides a visual environment to encourage the comparison of related "knowledge points" so as to foster meaningful learning, participants achieved significantly better learning achievement than those without the system support. In this research, a Knowledge Point (KP) is defined as "a minimum learning item which can independently describe the information of one certain piece of knowledge in a specific course"; a learner can understand a KP by its own expression or can acquire it by practice.

As mentioned before, in our previous experiment we encouraged the students to use comparison in their learning procedure. However, what do we mean by comparison exactly? "Comparison" is an essential skill required by critical thinking which involves logical thinking and reasoning. This skill refers to estimating, measuring, or noting the similarity or dissimilarity between two or more objects. The human thinking skills "compare" and "contrast" appear in both "comprehension" and "analysis" levels within the cognitive domain (in total six identified levels: "knowledge", "comprehension", "application", "analysis", "synthesis", and "evaluation") according to Bloom's taxonomy of intellectual behavior in learning, which contained three overlapping domains: the cognitive, psychomotor and affective domains [8]. From the educator's perspective, knowledge comparisons could significantly support learner comprehension of the new KP [9-11]. However, the existing e-learning systems, which organize the knowledge of a curriculum in a tree structure based on the textbook chapters or the class schedule, usually do not support the effective construction of knowledge frameworks by encouraging knowledge comparison because they cannot characterize essential relations between KPs. For example, in a Moodle system there are two KPs, " $\mathrm{d}$ " and " $\mathrm{t}$ ", which are located in Lesson 1 and Lesson 10, respectively. In Lesson 10, to compare the KP " $\mathrm{t}$ " with the prior $\mathrm{KP}$ " $\mathrm{d}$ ", the instructor has to indicate the location " $\mathrm{d}$ " (this can be done with a hyperlink) and explain the relation between " $\mathrm{d}$ " and " $\mathrm{t}$ ". Even so, it is still difficult for the learners to locate the learning materials which directly address the relation between these two KPs, unless they look through all the learning material in Lessons 1 and 10. The searching will be even more time-consuming if the learner is comparing three or more KPs in a course at one time.

To resolve these difficulties, an ontology-based Visualization Support System for e-book users (VSSE), which uses a hierarchical map structure to manage the KPs of a curriculum, was developed in this research to encourage the cultivation of comparison skills and foster meaningful learning.

\section{Visualization Supports for both Learners and Instructors}

\subsection{A Visualization Support for Learners Using E-Books}

For the learners in an e-book system, normally in one activity, they read several pages of a file. As shown in Figure 1, after one preview activity (for example, studying pages 10-13, which cover seven new knowledge points) in BookLooper, the learner can log in to VSSE, the Visualization Support System for E-book users, to check the new knowledge points just studied. Our system will try to encourage the learner to understand the relations between the new KPs visually. Furthermore, the system also will make use of the quiz results of learners to identify the learner's acquired KPs and then encourage the learner to compare the new KPs with related acquired ones visually. Finally, the system is also expected to recommend one KP or some KPs based on individual knowledge structure and guide learners to personalized learning paths. With this kind of visualization support, learners using BookLooper are expected to build up their knowledge framework more effectively. 


\section{Learner - e-book user}

\section{BookLooper}
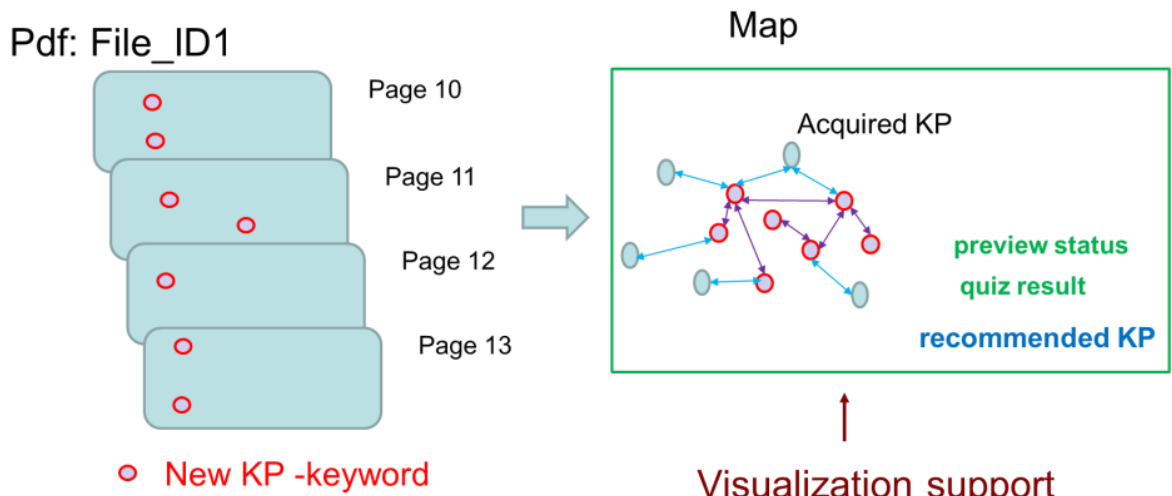

Figure 1. Visualization support for learners using e-books.

\subsection{A Visualization Support for Instructors of E-Book Users}

For instructor of e-book users, we also intend to provide several visualization supports. Firstly, the instructor can visually check all the learner's knowledge structures (including preview statuses and quiz results) through our systems. Secondly, the system will identify the most difficult KPs or relations based on the most frequently marked keywords and will highlight them to attract instructors' attention.

Most importantly, the system will make use of the quiz results of learners to identify the learners' acquired KPs or relations and then support the instructor in deciding group distribution for collaborative learning activities based on learners' knowledge structures. For example, as shown in Figure 2, assume an instructor of a given course plans to organize collaborative learning groups to study a new KP which has eight related KPs already taught in the course. To encourage the cooperation between learners in the same group, the system will identify Learners A, B and C, who acquired several $\mathrm{KPs}$ (overlap is allowed) related to the target KP, respectively, and recommend that the instructor put those three learners in one group. Otherwise, if learners in one group simply know the same related KPs, it is difficult for them to realize the rest of the related knowledge and reach the full understanding of the target KP effectively during the learning process. Based on this principle, the system will be designed to visually support instructors in deciding group distribution for collaborative learning activities.

\section{Instructor - e-book user}

\section{Group distribution for cooperative learning}

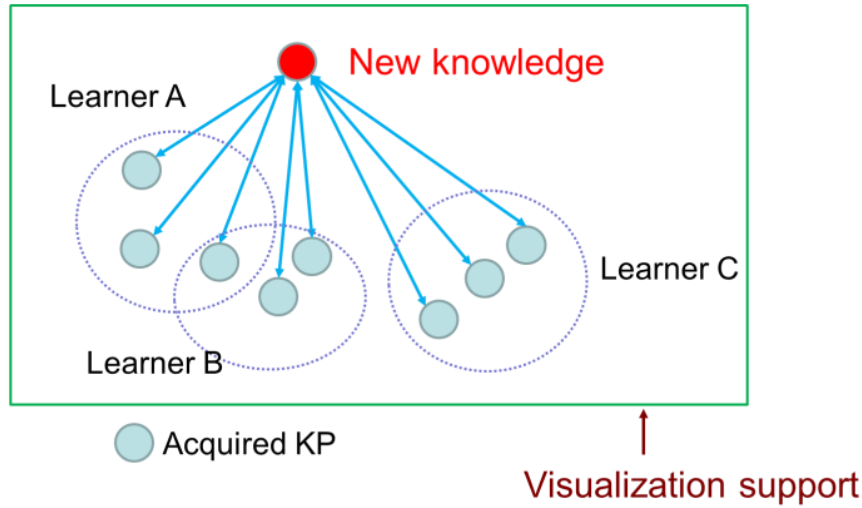

Figure 2. Visualization support for instructors of e-book users. 


\section{A Course-Centered Ontology for the Visualization Learning Support System}

\subsection{Semi-Automatically Built Course-Centered Ontology}

To facilitate the visualization supports mentioned in the previous section, the description of the information about all the KPs and the relations between KPs is required for the system. In this paper, we present a method to semi-automatically develop a course-centered ontology to describe all the required information from the knowledge in courses.

Firstly, we automatically exact the information from the "Syllabus system" of Kyushu University and create the basic framework of the course-centered ontology. As shown in Figure 3, for example, for the Kikan Education in the Arts and Science Department, there are 3730 courses registered in the syllabus system. For one course title, there are several courses taught by different professors or instructors; those courses may share some of the same keywords or have completely different keywords. One keyword also can be shared by courses which have different course titles. For example, "Differential equation" is taught in both "KIKAN Physics I A" and "Calculus and exercises" courses. Another typical example is "Entropy", which is not only taught in Thermodynamics courses but also in Information theory courses. In this step, the information of "course title/numbering code", "course code" and "keywords" from the syllabus are automatically exacted and used for the construction of the basic framework for ontology.

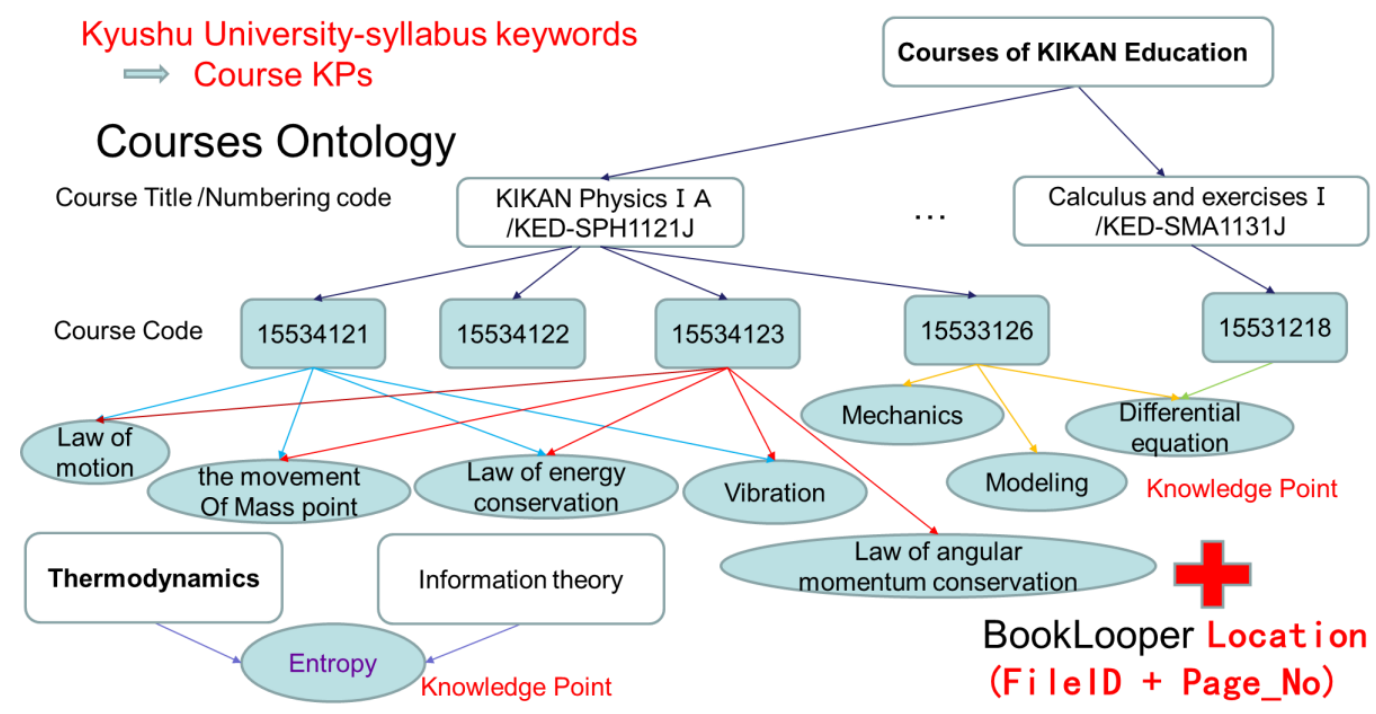

Figure 3. The basic framework of the course-centered ontology based on syllabus information.

However, for most of the courses in the syllabus system, less than 10 keywords are described; the basic ontology framework built on those syllabus keywords is sufficient to provide the visualization support described in the previous section. Therefore, in the second step, we encourage professors/instructors to manually modify the ontology using the 'Protégé' ontology editor [12] and then upload the modified ontology with the description of its related PDF file IDs in BookLooper.

For building a demo, we applied and adjusted the ontology design method described by Wang et al. [7] to develop a course-centered ontology of an existing computer science course, called course-centered ontology of computer science (COCS). We analyzed the learning materials of this computer science course, extracted about $100 \mathrm{KPs}$ and about 20 kinds of relations, and defined them in COCS. Figure 4 illustrates some KPs and their relations in COCS. We will describe the way in which COCS is designed and developed to show professors/instructors of other courses how to build up their course-centered ontology. Without doubt, how to combine all the course-centered ontology made by different professors/instructors into one ontology will be another issue we need to discuss in the future. 


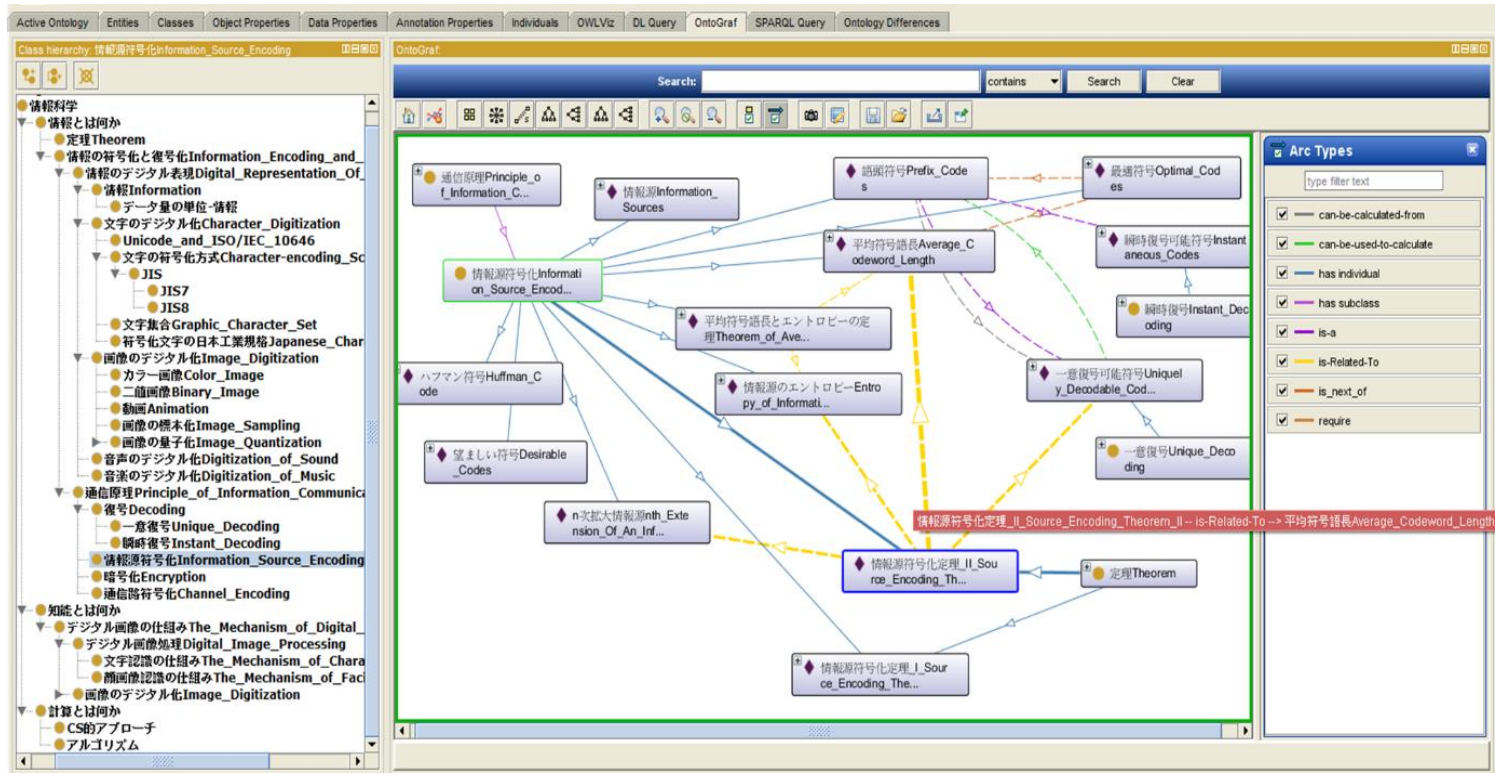

Figure 4. The course-centered ontology of computer science (COCS).

In the last step, we made a tool to automatically identify the location (including the file ID and the page number) of the KPs in the BookLooper system and put those location information details into the ontology.

Based on these three steps, the course-centered ontology will be developed semi-automatically. However, it is worth noting that maintaining this ontology is still time-consuming.

\subsection{A Visualization Learning Support System Providing a Knowledge Comparison Environment}

To automatically manipulate the course-centered ontology which describes all the relations between KPs, a system which is intended to provide visualization learning supports for the construction of learner knowledge frameworks is developed. Figure 5 shows a learner's view of the computer science course in the VSSE.

In the left part of this view, all the concepts of COCS are shown by a tree structure. Users can open all the concepts level by level until reaching the KP they are seeking. Further, a search function is also provided to the right, above the tree structure. The learner can set the time period (for example, from 3 January 2016 to 4 January 2016) and push the search button; then, the KPs which are involved in the pages that the learner reads during that time period will be highlighted. In addition, the learner can search items by keywords, and items which contain the keywords in the tree structure will also be highlighted to enable further checking.

When the user double-clicks one leaf which represents one $\mathrm{KP}$, the right relation panel will display this KP and all its related KPs lined by relations defined in COCS. For example, in Figure 5 the individual which represents the KP "shift_JIS" is chosen. Then users can get a visual representation of relevant information as shown in the relation panel.

Also, when the user moves the mouse on every node shown in the relation panel, the essential properties of that KP (represented by data properties of one individual in COCS) will be listed, while for every arc shown in the relations panel, the relation statement will be displayed (for example, the displayed relation axiom between "shift_JIS" and "JIS_X_0201" in Figure 5). Therefore, users can get the essential properties of every KP and all its related KPs from the relations panel conveniently. All this information is extracted automatically from the OWL (Web Ontology Language for Semantic Web, designed by W3C) [13] file of COCS. Furthermore, if there are too many relations shown in the relation panel, the user can select the relations of interest with the Arc Types panel. 


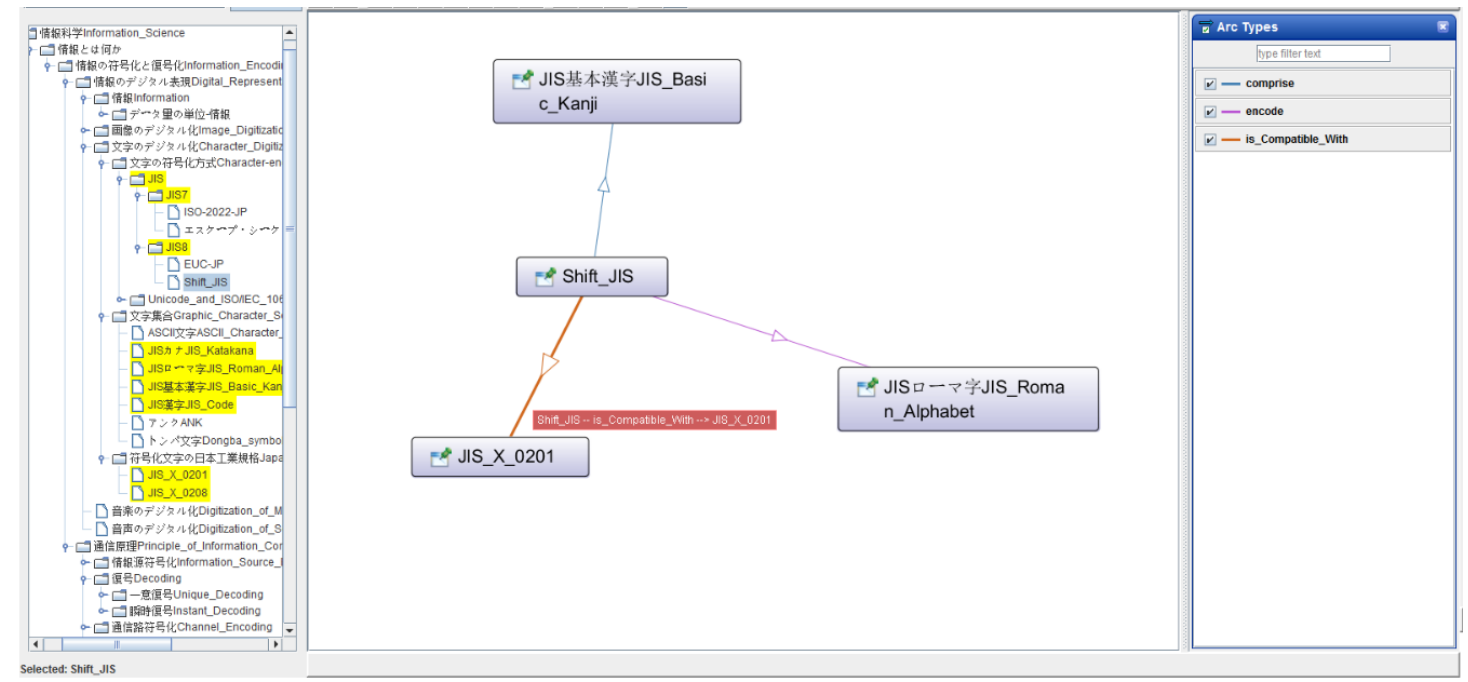

Figure 5. The main interface of VSSE for learners.

This system's function is expected to provide the visualization support for the construction of learner knowledge frameworks. The other function, which is intended to help instructors to understand the learners' knowledge structures and easily decide group distribution for collaborative learning activities, is still under development.

\section{Conclusions and Further Work}

In summary, we present a semi-automated method to construct a course-centered ontology, and based on this Course-centered Ontology we design and develop a system to provide visualization support for BookLooper e-book users. For learners of BookLooper users, the system is designed from the perspective of meaningful learning to visually support them to effectively construct their knowledge framework. For instructors of BookLooper users, when they are planning collaborative learning activities, the system can provide visualization support to help them decide the group distribution based on knowledge structure of learners.

After the development of these two mentioned functions, the visualization learning support system based on the ontology technique will be evaluated from various perspectives. In our further work, to encourage active engagement, a timely guided discovery learning environment [14] called "cache-cache comparison" [15] will be developed to encourage the learner to detect hidden relations between relevant KPs or hidden acquired KPs from given relevant KPs and relations through reflecting on the attributes of acquired knowledge visually. If learners keep making mistakes during the task the system will provide hints to help them rethink and get closer to the correct answer. The process of seeking hidden relations or concepts is intended to encourage learners to locate new knowledge in their knowledge structure and restructure existing knowledge; meanwhile, the iterative procedure of confirmation and modification in their own relation map ensures that they check the logical consistency of their ideas and clear up misunderstandings.

Acknowledgments: The research is supported by Kyushu University Interdisciplinary Programs in Education and Projects in Research Development, the Research and Development on Fundamental and Utilization Technologies for Social Big Data (No. 178A03), and the Commissioned Research of National Institute of Information and Communications Technology, Japan.

Author Contributions: Jingyun Wang and Hiroaki Ogata conceived and designed the system function; Jingyun Wang and Atsushi Shimada worked on the ontology design, and Atsushi Shimada provided the maintaining information for the ontology; Jingyun Wang performed the development of the ontology and the system and wrote the paper.

Conflicts of Interest: The authors declare no conflict of interest. 


\section{Abbreviations}

The following abbreviations are used in this manuscript:
KP Knowledge Point
VSSE visualization support system for E-book users

\section{References}

1. Yin, C.-J.; Okubo, F.; Shimada, A.; Oi, M.; Hirokawa, S.; Ogata, H. Identifying and Analyzing the Learning Behaviors of Students using e-Books. In Proceedings of the 23rd International Conference on Computers in Education (ICCE 2015), Hangzhou, China, 30 November-4 December 2015.

2. Ausubel, D.P. The Psychology of Meaningful Verbal Learning; Grune and Stratton: New York, NY, USA, 1963.

3. Ausubel, D.P. Educational Psychology: A Cognitive View; Holt: New York, NY, USA, 1968.

4. Ausubel, D.P.; Novak, J.D.; Hanesian, H. Educational Psychology: A Cognitive View, 2nd ed.; Holt, Rinehart and Winston: New York, NY, USA, 1978.

5. Novak, J.D. Meaningful learning: The essential factor for conceptual change in limited or appropriate propositional hierarchies (liphs) leading to empowerment of learners. Sci. Educ. 2002, 86, 548-571. [CrossRef]

6. Wang, J.; Mendori, T.; Xiong, J. A customizable language learning support system using ontology-driven engine. Int. J. Distance Educ. Technol. 2013, 11, 81-96. [CrossRef]

7. Wang, J.Y.; Mendori, T.; Juan, X. A Language Learning Support System Using Course-centered Ontology and Its Evaluation. Comput. Educ. 2014, 78, 278-293. [CrossRef]

8. Bloom, B.; Englehart, M.; Furst, E.; Hill, W.; Krathwohl, D. Taxonomy of Educational Objectives: The Classification of Educational Goals; Handbook I: Cognitive Domain; Longmans, Green: New York, NY, USA; Toronto, ON, Canada, 1956.

9. Fisher, K.M. The importance of prior knowledge in college science instruction. In Reform in Undergraduate Science Teaching for the 21st Century (Research in Science Education Series); Sunal, D.W., Wright, E.L., Bland, J., Eds.; Information Age Publishing Inc.: Greenwich, CT, USA, 2004; pp. 69-84.

10. Rittle-Johnson, B.; Star, Jon R.; Durkin, K. The importance of prior knowledge when comparing examples: Influences on conceptual and procedural knowledge of equation solving. J. Educ. Psychol. 2009, 101, 836-852. [CrossRef]

11. Amadieu, F.; Tricot, A.; Mariné, C. Interaction between prior knowledge and concept-map structure on hypertext comprehension, coherence of reading orders and disorientation. Interact. Comput. 2010, 22, 88-97. [CrossRef]

12. Horridge, M. A Practical Guide to Building OWL Ontologies Using Protégé 4 and CO-ODE Tools Edition 1.3. 2011. Available online: http://owl.cs.manchester.ac.uk/tutorials/protegeowltutorial/ (accessed on 10 March 2013).

13. W3C OWL Working Group. OWL 2 Web Ontology Language. 2012. Available online: http://www.w3.org/ TR/owl2-overview (accessed on 10 March 2016).

14. Bruner, J.S. The act of discovery. Harv. Educ. Rev. 1961, 31, 21-32.

15. Wang, J.Y.; Fujino, S. Cache-cache comparison for supporting meaningful learning. In Proceedings of the 12th International Conference on Cognition and Exploratory Learning (CELDA 2015), Maythoon, Ireland, 24-26 October 2015.

(C) 2016 by the authors; licensee MDPI, Basel, Switzerland. This article is an open access article distributed under the terms and conditions of the Creative Commons Attribution (CC-BY) license (http://creativecommons.org/licenses/by/4.0/). 